\title{
BIBECHANA
}

A Multidisciplinary Journal of Science, Technology and Mathematics ISSN 2091-0762 (Print), 2382-5340 (0nline)

Journal homepage: http://nepjol.info/index.php/BIBECHANA

Publisher: Research Council of Science and Technology, Biratnagar, Nepal

\section{First-principles study of structural, electronic and magnetic properties of Co-based quaternary Heusler compounds: CoFeCrAl, CoFeTiAs, CoFeCrGa and CoMnVAs}

\author{
Bikram Pandey $^{1}$, Ram Babu Ray ${ }^{2,5}$, Gopi Chandra Kaphle ${ }^{3,4,5, *}$ \\ ${ }^{1}$ Patan Multiple Campus, Tribhuwan University, Lalitpur, Nepal \\ ${ }^{2}$ Department of Physics, Amrit Science Campus, Tribhuwan University, Kathmandu, Nepal \\ ${ }^{3}$ Central Department of Physics Tribhuvan University, Kirtipur, Nepal \\ ${ }^{4}$ Condensed Matter Physics Research Center, Butwal, Nepal \\ ${ }^{5}$ Hydra Research and Policy Center, Kathmandu, Nepal \\ *Email: gck223@gmail.com
}

Article history: Received 21 September, 2017; Accepted 13 November, 2017

DOI: http://dx.doi.org/10.3126/bibechana.v15i0.18344

This work is licensed under the Creative Commons CC BY-NC License.

https://creativecommons.org/licenses/by-nc/4.0/

\section{c) (7) (8)}

Abstract

We study the structural, electronic and magnetic properties of Co-based LiMgPdSn-types of quaternary Heusler compounds (CoFeCrAl, CoFeTiAs, CoFeCrGa, and CoMnVAS) using Density Functional Theory (DFT) implemented on Tight Binding Linear Muffin-Tin Orbital within Atomic Sphere Approximation (TB-LMTO-ASA) Code. The optimized value of lattice parameter for $\mathrm{CoFeCrAl}$, CoFeTiAs, $\mathrm{CoFeCrGa}$ and CoMnVAs are found to be $5.61 \mathrm{~A}^{\circ}, 5.76 \mathrm{~A}^{\circ}, 5.61 \mathrm{~A}^{\circ}$ and $5.71 \mathrm{~A}^{\circ}$ , respectively. From the calculation of electronic band structure and spin polarized total density of states (DOS), we found that $\mathrm{CoFeCrAl}$ and $\mathrm{CoFeCrGa}$ are spin-gapless semiconductors with halfmetallic gap of $0.82 \mathrm{eV}$ and $0.25 \mathrm{eV}$ respectively. CoFeTiAs half-metal (Nearly spin-gapless semiconductor) with half-metallic gap of $0.38 \mathrm{eV}$, and CoMnVAs is found to be nearly gapless halfmetal. Magnetic moment of these compounds almost obey the Slater-Pauling rules. All these compounds expected to have high curie temperature which makes them significant for spintroincs/magnetoelectroincs applications.

Keywords: Spintronics; Quaternary Heusler compounds; Spin-gapless Semiconductor; Gapless-half metal, DFT.

\section{Introduction}

The major concern of material science is the search of materials and their properties that can be used to enhance the functionality of electronic devices. Conventionally electronic devices are based on the transformation of charge of electrons whereas in spintronics, the spin degree of freedom of electrons are added to the conventional charged based electronic devices to make them more enhanced. Spintronics is the recently burgeoning field, based on spin-polarized electron transport. It is believed that device based on spintronics can meet the necessities of higher aptitude of data storage, faster data transfer and faster data processing, through assemble of spins, and connection of electron spin to 
optics for future development of information and communication technology [1, 2]. Spin-gapless semiconductors (SGS) and Half-metallic (HM) materials are the new classes of materials that are characterized by $100 \%$ spin polarization. In Spin-gapless semiconductor, up spin band shows zero gap (gap-less) behavior at fermi-level, and down spin band exhibits semiconducting behavior with significant gap at fermi level. In SGSs, no energy is required to excite electrons from the valence band to the conduction band. In the case of half-metallic materials, one spin band shows metallic, while semiconducting for the other spin band. Both in SGS and HM, electrons as well as holes can be completely spin-polarized, which results in exceptional transport properties. Therefore, these materials utilize spin along with the charge of electrons to transmit current. Density of States n(E) as the function of energy (E) for spin gap-less semiconductors, half-metallic materials and gap-less halfmetals are shown in figure (1) $[3,4,5]$.

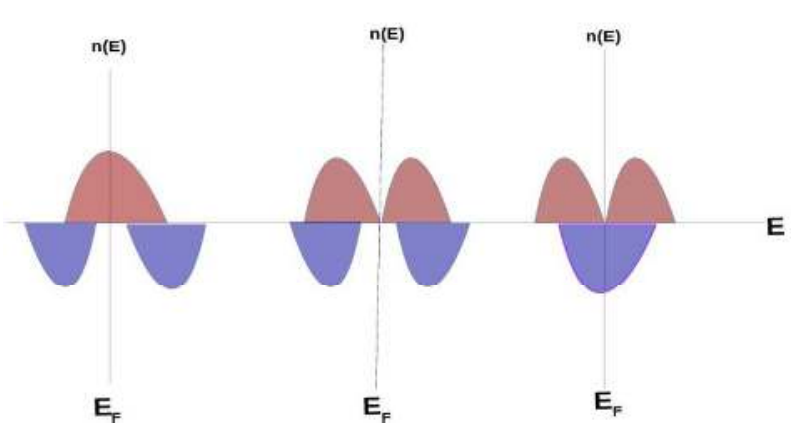

Fig. 1: (color online) Schematic representation of density of States $n(E)$ as the function of energy (E) showing half metal (left), spin-gapless semiconductor (center) and spin-gapless half metal (right). Red shaded area represents spin up states and blue shaded area represents spin down states.

Spintronics/Magnetoelectronics make some Heusler compounds momentous in the scientific research because, not only they bear spin-gapless semiconductor (SGS) and half-metalic properties but also the fundamental properties such as high curie temperature, spin polarization, generalized SlaterPauling rule atomic substitution etc.. Due to above mentioned reasons we are motivated to go more insight into the quaternary Heusler compounds. Generally, Quaternary Heusler compounds have the chemical formula XX'YZ, where $\mathrm{X}, \mathrm{X}$ (C) and $\mathrm{Y}$ are different transition metal atoms and $\mathrm{Z}$ is main group element. The valence of $Y$ is lower than the valence of the $X^{\prime}$ element, and the valence of the $X^{\prime}$ is lower than the valence of $X$ element. The sequence of the atoms along the face centered cubic (FCC) cube's diagonal is X-Y-X'-Z which is energetically the most stable [6, 7].

The concept of spin-gap less semiconductor was first proposed by Wang [3] in 2008, that doping of $\mathrm{PbPd} \mathrm{O}_{2}$, gap-less semiconductor with transition metal atoms would lead to a SGS based on bandstructure calculations. Later, Kim et al. and Choo et al. confirmed this experimentally the electronic structure of $\mathrm{Mn}$ and $\mathrm{Co}$ doped $\mathrm{PbPd} \mathrm{O}_{2}$ polycrystalline films[8]. Several Heusler compounds have been reported to be a spin-gap less semiconductor. Among the quaternary Heusler (LiMgPdSn-type) compounds that were studied several have been reported as the half-metal and spin-gap less semiconductors. Bainsla et al. studied the equiatomic quaternary Heusler compound $\mathrm{CoFeCrGa}$, from first-principles calculation, and confirmed its SGS nature experimentally [9]. In 2013, Xu et al. examined the band structure of the quaternary Heusler compound CoFeMnSi from of first-principles calculation and predicted as the SGS, which was confirmed experimentally by Bainsla et al. in 2015 $[9,10]$.

The connection between magnetic and electronic properties of half-metallic Heusler compounds are given by Slater-Pauling rule. For the half-metallic full-Heusler compounds Slater-Pauling rule is 
given by $\mathrm{M}_{\mathrm{t}}=Z_{\mathrm{t}}-24$, where $M_{t}$ is the total spin magnetic moment of unit cell in $\mu_{B}$ and $Z_{\mathrm{t}}$ is the total valence electrons [11]. Most of the LiMgPdSn-type quaternary Heusler compounds which obey same Slater-Pauling rule for full-Heusler compounds were half metals. In order to explain the origin of half metallic gap and Slater-Pauling behavior of full-Heusler compounds, there have been detailed analysis of hybridization scheme, which is same for the quaternary Heusler compounds [12]. In this communication, we are focusing towards some Co-based quaternary LiMgPdSn-types Heusler compounds having 26 valence electrons in the search of SGS and to go more insight into their properties using TB-LMTO-ASA. This is because Co based compounds show comparatively high curie temperature and high spin polarization basicaly used for GMR , TMR etc. and modern technological devices. In addition, some earlier researcher reported a semi-empirical rule of valence electrons being 26 or 28 for the sake of searching for SGS among Heusler compounds from the comprehensive study of atomic and orbital hybridization scheme. We believe that present work will assist to guide the scope of finding SGS, among LiMgPdSn-type quaternary Heusler compounds having 26 (or 28) valence electrons in the future. Number of works have been carried out using TBLMTO-ASA approach in ordered binary alloys [13-14], disordered alloys [15], perovskites [1617] and Heusler alloys [18]. The other motivation or aim is to use TBLMTO-ASA approach on quaternary alloys.

This article is organized as follows, section 2 discusses the method and computational details, followed by results and discussion. Section 3 contains conclusions followed by the references listed at the end of section after the acknowledgments.

\section{Methodology and Computational Details}

We performed all the calculation using first principles based density functional theory (DFT) implemented on Tight Binding Linear Muffin-Tin Orbital within Atomic Sphere Approximation (TBLMTO-ASA) code [19-20]. The partial-potential linearized augmented plane wave is used within atomic spheres which ultimately gives total potential through tail cancellation process [21-22]. Minimal basis set parameter is used throughout the calculations. The electronic exchange-correlation function we used is the local density approximation (LDA). The LDA+U is also implemented whenever we required [23-25] for the calculations. The $8 \times 8 \times 8$ numbers of $\mathrm{k}$ points meshes are considered for the Brillouin zone integration. The core states are taken relativistic where as valence states are taken as semi relativistic nature. The self-consistency calculations are considered to be succeeded when the total energy difference between consecutive iterations is less than $10^{-6}$ Ryd. per formula unit.

\section{Results and Discussion}

\subsection{Structural Stability Analysis}

Depending upon the possible site of occupation of Y on X, X', Y, and Z, Quaternary Heusler alloys with space group F-43 (No. 216) have categorized into Y1, Y2 and Y3-types of structures (collectively named as Y-types of structures) [26]. The energy minimization process is carried out, from the spin-polarized energies as the function of lattice parameter, for the all possible Y-type of structures to find out most stable structure. We found, from the calculation that the most stable equilibrium structure is Y1-type, with atom X, X', Y and Z locate the position at (0.75 0.75075$),(0.25$ $0.250 .25),\left(\begin{array}{lll}0.5 & 0.5 & 0.5\end{array}\right)$ and $\left(\begin{array}{lll}0 & 0 & 0\end{array}\right)$ respectively, shown in figure (1). The spin polarized total energies as the function of lattice parameters for the different possible Y-type structure of $\mathrm{CoFeCrAl}$ are shown in figure (2), which indicate that Y1-type crystal structure is most stable and similar results are obtained for CoFeTiAs, CoFeCrGa and CoMnVAs respectively. The optimized value of lattice parameter for CoFeCrAl, CoFeTiAs, CoFeCrGa and CoMnVAs are found to be $5.61 \mathrm{~A}^{\circ}, 5.76 \mathrm{~A}^{\circ}$, $5.61 \mathrm{~A}^{\circ}$ and $5.71 \mathrm{~A}^{\circ}$ respectively.

\subsection{Electronic band structure and Density of states}

The spin polarized density of states (DOS) for CoFeCrAl, CoFeCrGa CoFeTiAs and CoMnVAs at their most stable equilibrium lattice constant are shown in figure (3). Figures show that $\mathrm{CoFeCrAl}$ and 
CoFeCrGa are spin-gap less semiconductors. Since, these compounds exhibit the finite energy gap at fermi level in spin-down channel, while the fermi level falls within a zero-energy gap in the spin-up channel. Furthermore, the extensive studied of the band structure also confirmed it. In the band structure of $\mathrm{CoFeCrAl}$ for spin up channel, it is seen that bands just touches the fermi-level at symmetric points $\mathrm{X}$ and between $\mathrm{K}$ and $\Gamma$ in conduction band, while band touches fermi level at symmetric points $\mathrm{L}$ and $\mathrm{W}$ in valence band, and no bands crossing the fermi level. In spin down channel there is $0.82 \mathrm{eV}$ band gap between conduction band and valence band near the fermi level as shown in figure. This confirmed the SGS nature of CoFeCrAl. The gap less behavior in spin up band and semiconducting behavior in spin down band, clearly indicates that CoFeCrAl is SGS material. Present calculations also support the results obtained by $\mathrm{Xu}$ et al. and $\mathrm{K}$. ozdogan et al. $[10,12]$ they predicted $\mathrm{CoFeCrAl}$ as SGS material. In case of $\mathrm{CoFeCrGa}$, band just touches the fermi-level at symmetric points $\mathrm{W}$ and $\mathrm{K}$ in the conduction band while band touches the fermi-level at symmetric points $\mathrm{L}$ in the valence band for spin up band structure and in the spin down band structure, there is $0.25 \mathrm{eV}$ band gap, indicating that $\mathrm{CoFeCrGa}$ is $\mathrm{SGS}$ material as shown in figure. This calculation also support the results obtained by Bainsla et al. who predicted CoFeCrGa as SGS from first-principles calculations, and verified experimentally $[9,10]$.
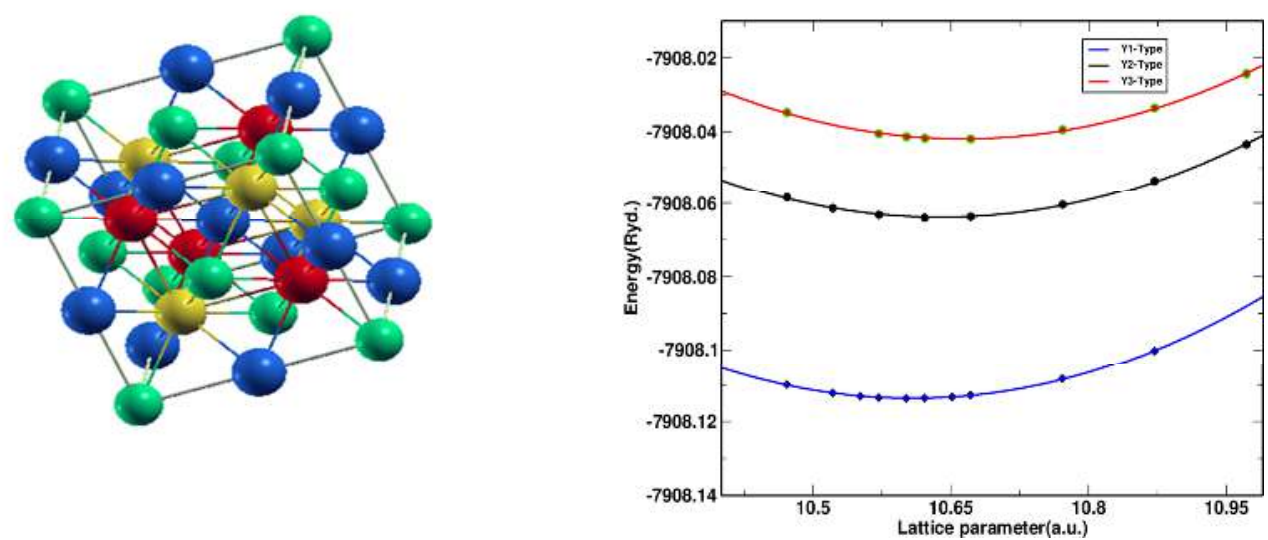

Fig. 2.: (color online) (a) Y1-type of structure for XX'YZ Heusler compound, red, blue, yellow and green color represents $\mathrm{X}, \mathrm{X}$,, $\mathrm{Y}$ and $\mathrm{Z}$ atom respectively. (b) The spin polarized energies as a function of lattice parameter for the different possible Y-types of structure for $\mathrm{CoFeCrAl}$.

The spin polarized density of states (DOS) indicates that CoFeTiAs, is half-metal, since the continuous but low DOS at the fermi level of spin up channel shows the usual metallic behavior, meanwhile the spin down channel exhibits a semiconducting nature with a gap of $0.38 \mathrm{eV}$ at the fermi level. K. ozdogan et al. [12] also studied the CoFeTiAs using FPLO method and reported as SGS. The half-metallic nature of CoFeTiAs has also been considered by L. Xiong et al. [27]. Although, semiconducting gap reported by them is $0.18 \mathrm{eV}$. It is believed that the contrast arises from the different electronic band structure calculation methods used in the two studies. The study of spin band structure in detail reveals that a small overlap of band around the fermi level although bands does not cross with each other as shown figure (4c), thus we can conclude that CoFeTiAs, is half-metal also possible candidate for SGS i.e. nearly spin-gap less semiconductor.

The spin polarized total density of states (DOS) indicate that CoMnVAs is a gap less-metallic nature since it shows that almost vanishing of DOS at fermi level in spin up channel and continuous DOS at fermi level in spin down channel. On the other hand, the study of spin up band structure of CoMnVAs in detail shows that there is a small overlay of band at fermi level in conduction band but 
do not crossing with valence band which is shown in figure (4d), on this base we can say that CoMnVAs is nearly gap less-half metal.

Present system consists of three transition metals and one main group element (sp element). The study of atom resolved and orbital resolved partial density of states (PDOS) shows that the major contribution in DOS is due to the d-orbital of transition metals and the contribution of main group element is almost negligible as shown in figure ( $3 \mathrm{c}$ and $3 \mathrm{~d})$.
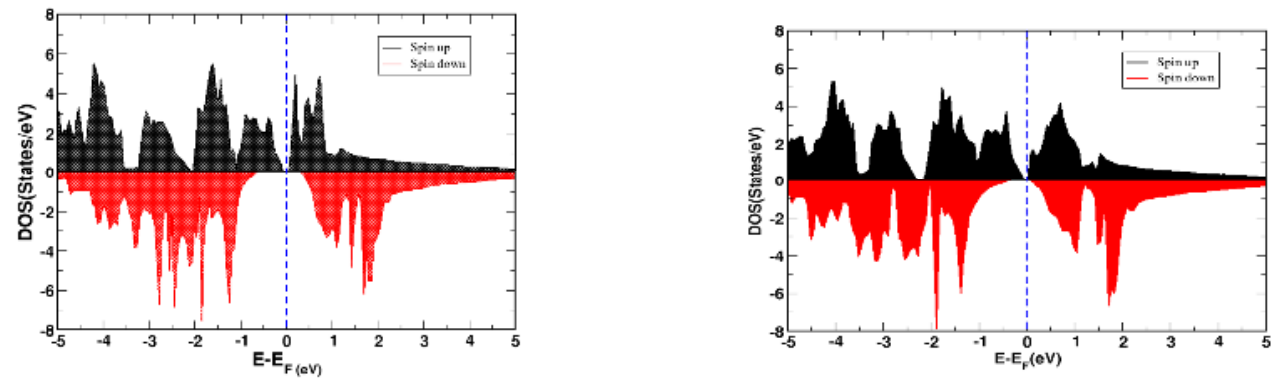

Fig (3a): (color online) Density of states (DOS) of CoFeCrAl (left) and CoFeCrGa (right).
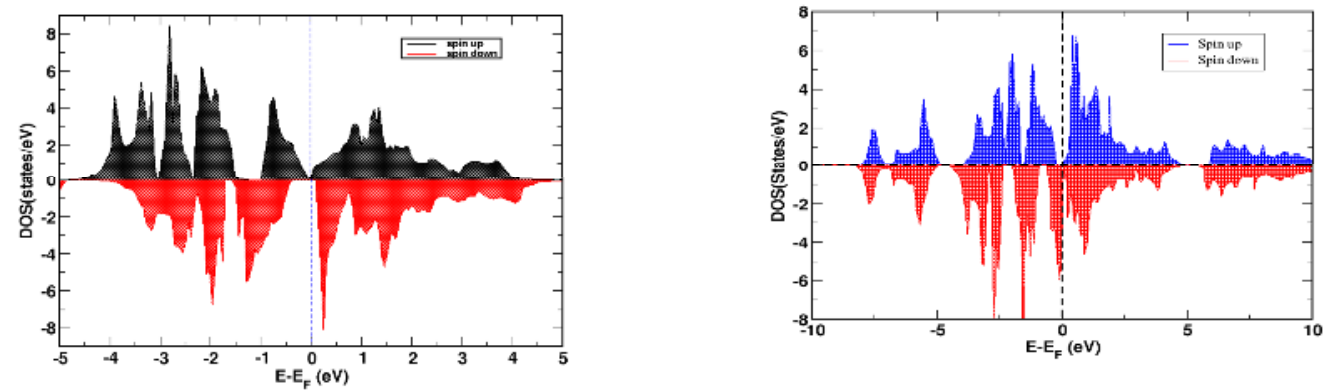

Fig. (3b): (color online) Density of states (DOS) of CoFeTiAs (left) and CoMnVAs (right) .
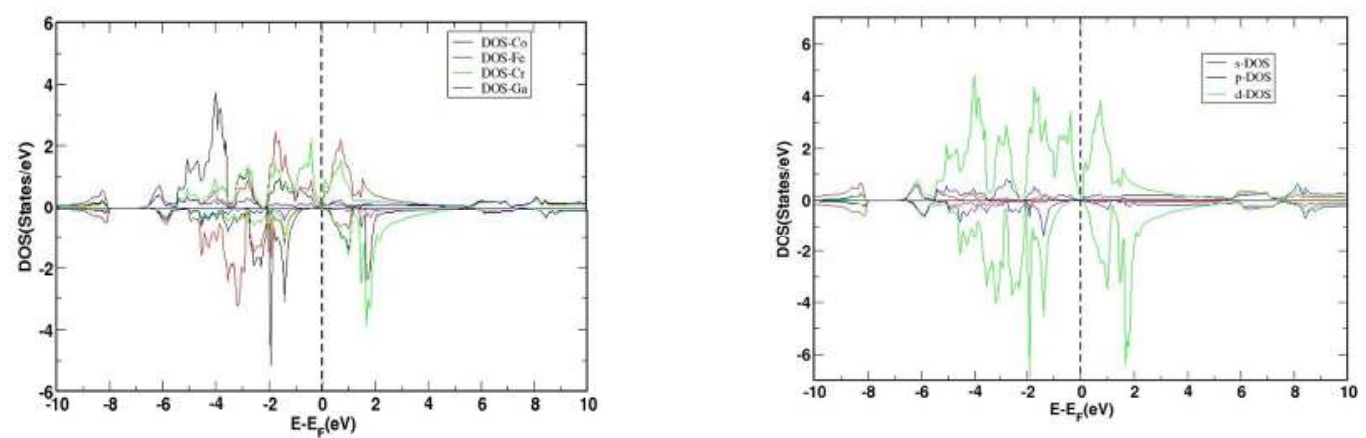

Fig. (3c): (color online) Atom resolved (left) and orbital resolved (right) partial DOS of CoFeCrGa. 

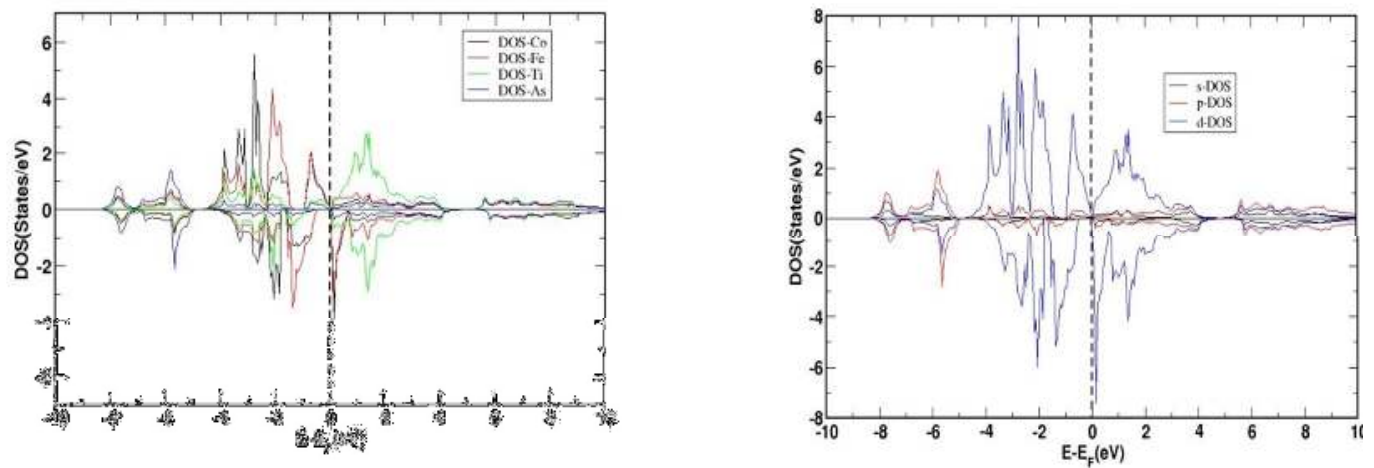

Fig. (3d): (color online) Atom resolved (left) and orbital resolved (right) partial DOS of CoFeTiAs.
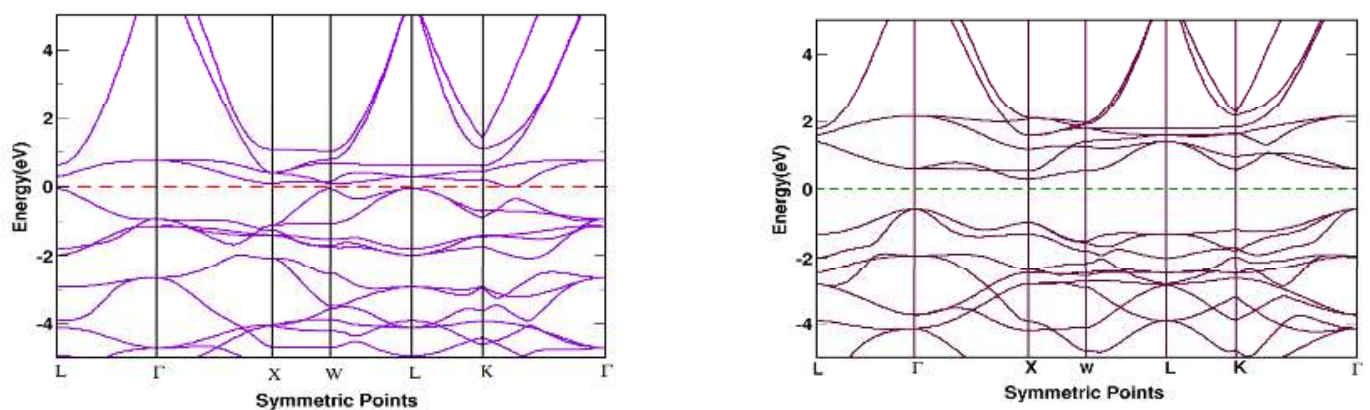

Fig.(4a): (color online) Spin up (left) and spin down (right) band structure of CoFeCrAl.
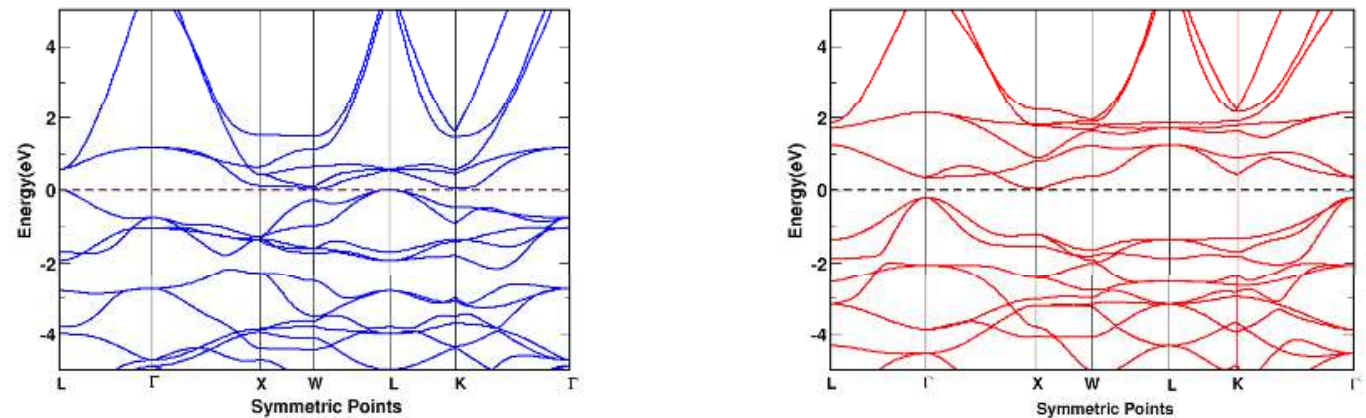

Fig. (4b): (color online) Spin up (left) and spin down (right) band structure of $\mathrm{CoFeCrGa}$. 

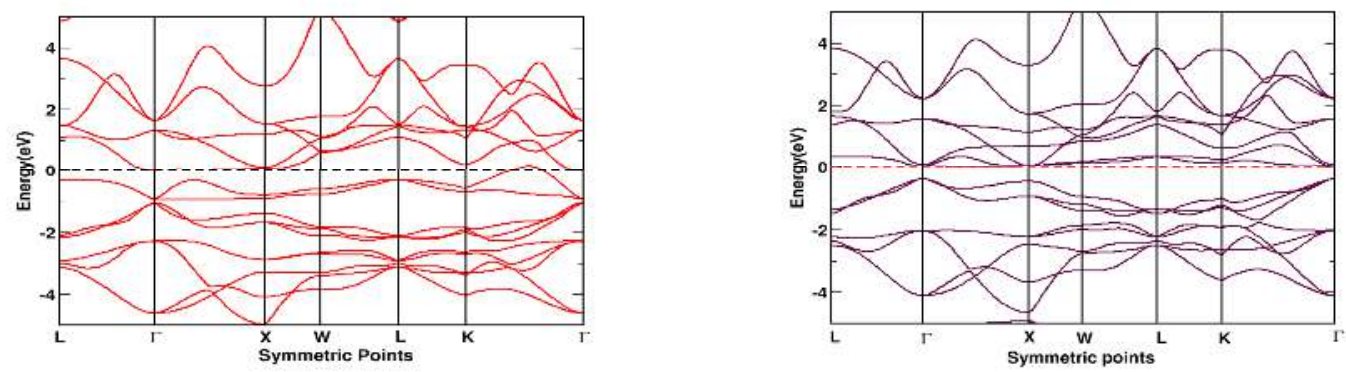

Fig. (4c): (color online) Spin up (left) and spin down (right) band structure of CoFeTiAs.
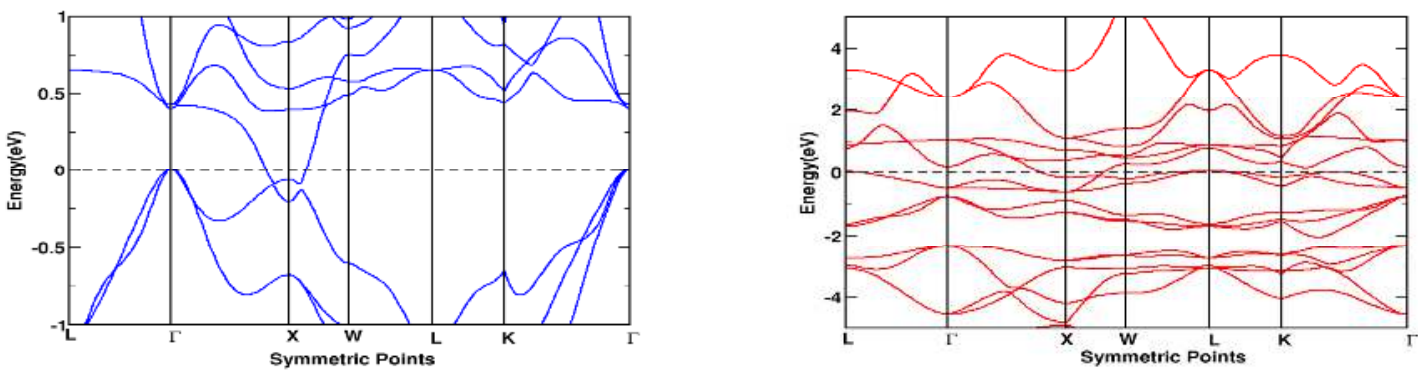

Fig. (4d): (color online) Spin up (left) and spin down (right) band structure of CoMnVAs.

\subsection{Magnetic moments}

The unsymmetrical density of states (DOS) for spin up and spin down channel clearly indicate that systems under the present study are magnetic materials. The total magnetic moment $\left(\mathrm{M}^{\mathrm{T}}\right)$ of LiMgSnPd-type quaternary Heusler compounds (CoFeCrAl, CoFeTiAs, CoFeCrGa and CoMnVAs) at their optimized lattice parameter that we calculated are shown in table (1). All the compounds have 26 total number of valence electrons $\left(Z_{\mathrm{t}}\right)$ and calculated magnetic moments almost follow the Slater Pauling rule $\mathrm{M}_{\mathrm{T}}=Z_{\mathrm{t}}-24$. In order to explain the origin of half metallic gap and Slater-Pauling behavior of full-Heusler compounds, there have been detailed analysis of hybridization scheme, which is same for the quaternary heusler (LiMgPdSn-type) compounds. The spin-gapless semiconductor, having 26 total number of valence electrons, schematic representation of the energy levels of the spin up and spin down electronic band structure done by K. ozdogan et al. [12] is shown figure (5).

Table [1]: The calculated equilibrium lattice constant (a), the total spin magnetic moment $\left(\mathrm{m}^{\mathrm{T}}\right)$ in $\mu_{\mathrm{B}}$ and atom resolved magnetic moment with total number of valence electron $\left(Z_{t}\right)$ of LiMgSnPd-type Heusler compounds (CoFeCrAl, CoFeTiAs CoFeCrGa and CoMnVAs).

\begin{tabular}{|l|l|l|l|l|l|l|l|}
\hline $\mathbf{X X} \mathbf{Y} \mathbf{Z}$ & $\mathbf{a}^{\prime} \mathbf{A}^{\circ} \mathbf{)}$ & $\mathbf{M}^{\mathbf{X}}$ & $\mathbf{M}^{\mathbf{X}^{\prime}}$ & $\mathbf{M}^{\mathbf{Y}}$ & $\mathbf{M}^{\mathbf{Z}}$ & $\mathbf{M}^{\mathbf{T}}\left(\boldsymbol{\mu}_{\mathbf{B}} \mathbf{}^{\mathbf{2}}\right.$ & $\mathbf{Z}_{\mathbf{T}}$ \\
\hline CoFeCrAl & 5.61 & 0.885 & -0.076 & 1.233 & -0.049 & 1.993 & 26 \\
\hline CoFeTiAs & 5.76 & 1.048 & 1.031 & -0.121 & 0.040 & 1.999 & 26 \\
\hline CoFeCrGa & 5.61 & 0.841 & -0.132 & 1.331 & -0.039 & 2.002 & 26 \\
\hline CoMnVAs & 5.71 & 1.151 & 0.444 & 0.376 & 0.019 & 1.986 & 26 \\
\hline
\end{tabular}




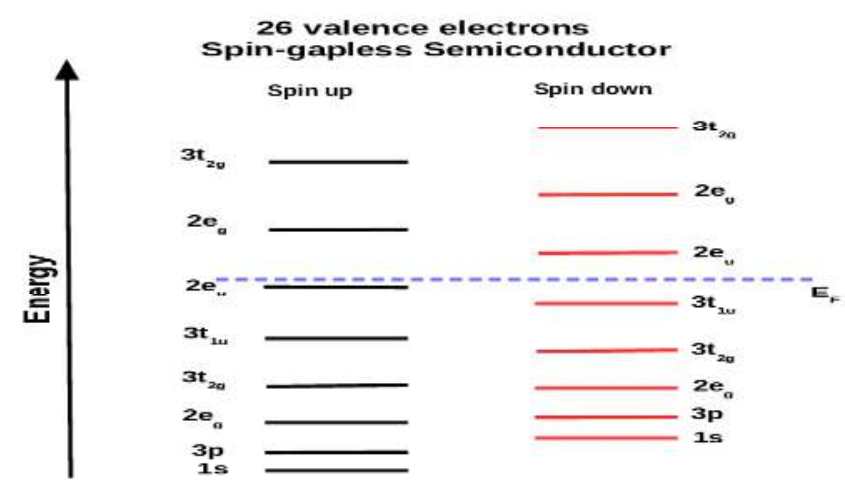

Fig. (5): (color online) Schematic representation of the energy levels of the up and down spin electronic band structure for the 26 valence-electrons spin-gapless semiconductors.

\subsection{Charge Distribution and Hartree Potential}

We plot the hartree potential of $\mathrm{CoFeCrAl}$, CoFeTiAs $\mathrm{CoFeCrGa}$ and CoMnVAs using their space group F-43m (no. 216) at their optimized value of lattice parameter, which support the theory that considered the muffin-tin potential in which the potential is maximum near the core of the atom and almost flat in the interstitial space region as shown in figure (6). This indicates that potential is sharp where the charge distribution is maximum and flat at the interstitial regions. The charge density distribution plot also supports the same.
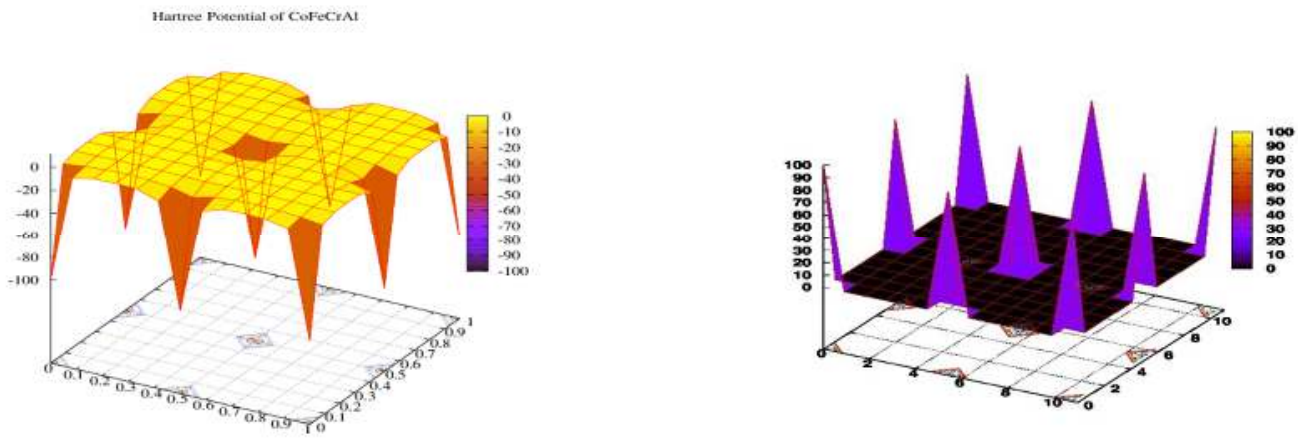

Fig. ( 6): (color online) Hartree Potential (left) and Charge density distribution (Right) of CoFeTiAs.

\section{Conclusions}

In conclusion, we study the structural, electronic and magnetic properties of $\mathrm{CoFeCrAl}$, CoFeTiAs, $\mathrm{CoFeCrGa}$ and CoMnVAs, using TB- LMTO-ASA based on density functional theory. First, we have found that Y1-type structure is most stable structure among the possible Y-types of structures through energy minimization process. Next, from the detailed study of electronic band structure and spin polarized DOS, we have found that $\mathrm{CoFeCrAl}, \mathrm{CoFeCrGa}$ are SGS, with half-metallic band gap of 
$0.82 \mathrm{eV}$ and $0.25 \mathrm{eV}$, respectively. The CoFeTiAs is found to be nearly spin-gapless semiconductor, with half-metallic gap $0.38 \mathrm{eV}$, also possible candidate for SGS, and CoMnVAs is nearly gapless-halfmetal. We have also calculated the magnetic moment and found that all the compounds under present study almost follow the Slater-Pauling rule. Finally, we can conclude that all the Heusler compound having 26 valence electrons are not SGS. The semi-empirical rule with 26 valence electrons can play a supportive for the possibility of finding SGS in future, which is one of the necessary but not sufficient conditions.

\section{Acknowledgments}

This work is partially supported by HRPCN. The authors would like to express gratitude to Prof. N. P. Adhikari, CDP, TU, Kirtipur, Kathmandu, Nepal and Prof. A. Mookerjee, SNBNCBS, Kolkota, India for providing TB-LMTO-ASA package. One of the author B. Pandey would like to thank Physics for Curious Brain Nepal, a research group, for the valuable discussions during this work.

\section{References}

[1] A. Hirohata and K. Tankanashi, Future perspectives for spintronic devices, J. Phys. D: Applied. Phys. 47(19) (2014) 193001-40. doi.org/10.1088/0022-3727/47/19/193001.

[2] C. Felser and A. Hirohata, Editors, Heusler Alloys: Properties, Growth, Applications, Springer Series in Material Science 222, London, 2016.

[3] X. L. Wang, Proposal for a New Class of Materials: Spin Gapless Semiconductors Phys. Rev Lett 100(15) (2008) 156404-1- 156404-4. doi.org/10.1103/PhysRevLett.100.156404.

[4] M. Isaak , J. Tsidilkovski, Edited by K. von Klitzing, R. Merlin, H. J. Queisser, B. Keimer, Springer Series in Solid-State Sciences, Vol. 116, London, 1996.

[5] R. A. de Groot and F. M. Mueller, New Class of Materials: Half-Metallic Ferromagnets, Phys. Rev. Lett 50 (25),(1983) 2024-2027 . doi.org/10.1103/PhysRevLett.50.2024.

[6] X. F. Dai, G. D. Liu, G. H. Fecher, C. Felser, Y. X. Li and H. Y. Liu, New quarternary half metallic material CoFeMnSi, J. Appl. Phys. 105 (2009) 07E901. doi.org/10.1063/1.3062812.

[7] V. Alijani, J. Winterlik, G. H. Fecher, S. S. Naghavi and C. Felser, Quaternary half-metallic Heusler ferromagnets for spintronics applications, Phys. Rev. B 83 (2011) 184428. doi.org/10.1103/PhysRevB.83.184428.

[8] D. H. Kim, J. Hwang, E. Leek, K. J. Lee, S. M. Choo, et al., Valence states and electronic structures of Co and $\mathrm{Mn}$ substituted spin gapless semiconductor $\mathrm{PbPdO}_{2}$, Appl. Phys. Lett. 104 (2) (2014) 022411.

doi.org/10.1063/1.4861883.

[9] L. Bainsla , A. I. Mallick, M. M. Raja, A. A. Coelho, A. K. Nigam, et al., Origin of spin gapless semiconductor behavior in CoFeCrGa: Theory and Experiment, Phys. Rev. B 92, (2015) 045201. doi.org/10.1103/PhysRevB.92.045201.

[10] G. Z. Xu, E. K. Liu, Y. Du, G. J. Li, G. D. Liu, et al., A new spin gapless semiconductors family: Quaternary Heusler compounds, Euro. Phys. Lett. 102 (1) (2013) 17007. doi.org/10.1209/0295- 5075/102/17007.

[11] I. Galanakis, P. H. Dederichs, N. Papanikolau.,Slater-Pauling behavior and origin of the half-metallicity of the full-Heusler alloys, Phys. Rev. B 66 (2002)174429-1- 174429-9. doi.org/10.1103/PhysRevB.66.174429.

[12] K. Özdoğan, E. Şaşıoğlu, and I. Galanakis, Slater-Pauling behavior in LiMgPdSn-type multifunctional quaternary Heusler materials: Half-metallicity, spin-gapless and magnetic semiconductors, J. Appl. Phys. 113 (19),(2013) 193903. doi.org/10.1063/1.4805063.

[13] S. Pandey, G. C. Kaphle and N. P. Adhikari, Electronic structure and magnetic properties of bulk elements (Fe and $\mathrm{Pd}$ ) and ordered binary alloys (FePd and Fe3Pd):TB-LMTO-ASA approach BIBECHANA 11 (2014) 60-69. doi.org/10.3126/bibechana.v11i0.10381. 
[14] R. P. Sedhain, G. C. Kaphle, Structural and Electronic Properties of Transition Metal Di-Chalcogenides (MX2) $\mathrm{M}=(\mathrm{Mo}, \mathrm{W})$ and $\mathrm{X}=(\mathrm{S}, \mathrm{Se})$ in Bulk State: A First-Principles Study, Journal of Institute of Science and Technology 22(1) (2017) 41-50. doi.org/10.3126/jist.v22i1.17738.

[15] G. C. Kaphle, S., Ganguly, R. Banerjee, R. Khanal, C. M. Adhikari, N. P. Adhikari, and A. Mookerjee, A study of magnetism in disordered $\mathrm{Pt}-\mathrm{Mn}, \mathrm{Pd}-\mathrm{Mn}$ and $\mathrm{Ni}-\mathrm{Mn}$ alloys: an augmented space recursion approach, Journal of Physics: Condensed Matte, 24, 295501(2012). doi:10.1088/0953-8984/24/29/295501-1-295501-12.

[16] S. Lamichhane, B. Aryal, G. C. Kaphle, N. P. Adhikari, Structural and electronic properties of perovskite hydrides AcaH3 (A=Cs and Rb), BIBECHANA 13 (2014) 94-99. doi.org/10.3126/bibechana.v13i0.13437.

[17] M. P. Ghimire, G. C. Kaphle, R. K. Thapa,Electronic and Magnetic Properties of Double Perovskites Nd2MgIrO6, Journal of Nepal Physical Society 3(1) (2016) 50-54.

: doi.org/10.3126/jnphyssoc.v3i1.14442.

[18] P. Sharma, G. C. Kaphle, Electronic and Magnetic Properties of Half Metallic Heusler Alloy Co2MnSi: A First-Principles Study, Journal of Nepal Physical Society 4(1) (2017) 60-66.

: doi.org/10.3126/jnphyssoc.v4i1.17338.

[19] W. Kohn and L. J. Sham, Self-Consistent Equations Including Exchange and Correlation Effects, Phys. Rev. 140 (1965) A1133-A1138. doi.org/10.1103/PhysRev.140.A1133.

[20] H. L. Skriver, The LMTO Method, Springer-Verlag, Berlin, Germany, 1984.

[21] O. K. Andersen and O. Jepsen, Explicit, First-Principles Tight-Binding Theory, Phys. Rev. Lett. 53 (1984) 2571.doi.org/10.1103/PhysRevLett.53.2571.

[22] M. Zwierzycki and O. K. Andersen, The Overlapping Muffin-Tin Approximation, Acta Physica Polonia A 115(1), (2009) 64-68. doi.org/10.12693/APhysPolA.115.64.

[23] V. I. Anisimov, F. Aryasetiawan and A. I. Lichtenstein, First-principles calculations of the electronic structure and spectra of strongly correlated systems: the LDA + U method, J. Phys.: Condens. Matter 9 (1997) 767-80. doi.org/10.1088/0953-8984/9/4/002.

[24] I. V. Solovyev, P. H. Dederichs and V. I. Anisimov, Corrected atomic limit in the local-density approximation and the electronic structure of d impurities in Rb, Phys. Rev. B 50 (1994) 6861. doi.org/10.1103/PhysRevB.50.16861.

[25] R. M. Martin, Electronic Structure: Basic Theory and Practical Methods, Cambridge University Press, U. K., 2004.

[26] V. Alijani, S. Ouardi, G. H. Fescher, J. Wintwrlik, S. S. Naghavi et al., Electronic, structural, and magnetic properties of the half-metallic ferromagnetic quaternary Heusler compounds $\mathrm{CoFeMnZ}(\mathrm{Z}=$ Al, Ga, Si, Ge), Phys. Rev. B 84 (2011) 224416-1-224416-10. doi.org/10.1103/PhysRevB.84.224416.

[27] L. Xiong, L. Yi, G. Y. Gao,Search for half-metallic magnets with large half-metallic gaps in the quaternary Heusler alloys CoFeTiZ and $\mathrm{CoFeVZ}(\mathrm{Z}=\mathrm{Al}, \mathrm{Ga}, \mathrm{Si}, \mathrm{Ge}, \mathrm{Sb})$, J. Magn. Magn. Mater. 360,(2014) 98-103. doi.org/10.1016/j.jmmm.2014.02.050. 